\title{
A utilização de tecnologias da informação em saúde para o enfrentamento da pandemia do Covid-19 no Brasil
}

The use of health information technologies to face the Covid-19 pandemic in Brazil

El uso de tecnologías de la información en salud para enfrentar la pandemia Covid-19 en Brasil

\author{
Akeni Lobo Coelho ${ }^{1}$ \\ Indyara de Araujo Morais ${ }^{2}$ \\ Weverton Vieira da Silva Rosa ${ }^{3}$
}

\section{Resumo}

Objetivos: demonstrar como o uso de tecnologias da informação em saúde, por meio do monitoramento de redes de acesso público, contribui na redução de agravos das condições de saúde em pandemias, avaliando o uso das tecnologias nas experiências internacionais e sua potencial aplicação à realidade brasileira. Metodologia: foi realizada revisão integrativa com levantamento de artigos nas bases de dados Scielo, Pubmed e Google Acadêmico. Foram selecionados 21 artigos e 11 documentos diversos, como peças jornalísticas, capítulos de livros, resenhas, entre outros. Os artigos passaram por leitura e análise crítica por dois pesquisadores distintos. Um terceiro pesquisador foi responsável pela interpretação dos resultados. Resultados: o estudo demonstrou que existe uma grande tendência mundial para o uso de tecnologias da informação e comunicação como ferramentas no auxílio ao enfrentamento de pandemias, visto que foram diversos os exemplos mundiais que trataram da utilização das tecnologias para o controle do Covid-19, e esse uso depende de uma evolução e de aprimoramento das ferramentas tecnológicas e das legislações envolvidas. No Brasil, existe um grande potencial para evolução nessa área, com o aumento da captação e utilização dos dados, auxiliando nas estratégias e ações na saúde pública. Porém, em todos os cenários apresentados, faz-se necessário aprimoramento e adequações no âmbito legislativo - conferindo maior responsabilidade legal aos direitos de privacidade - e social, com a ampliação da infraestrutura da internet, alcançando toda população e, principalmente, com a maior confiabilidade dos dados. Conclusão: a partir do entendimento sobre o cenário de utilização das Tecnologias da Informação e Comunicação em diversos países e como foram às respostas nos respectivos territórios, foi feita uma comparação com o Brasil e percebeu-se que a capacidade tecnológica brasileira é limitada de uma forma geral. As questões socioculturais no Brasil se destacam, por se tratarem, por vezes, de barreiras existentes no cenário brasileiro, e que devem ser discutidas, visando um melhor entendimento sobre o uso dessas tecnologias nas políticas públicas de forma a alcançar toda população. Com isso, fora visto que é necessária a participação consciente e ativa dos

\footnotetext{
${ }^{1}$ Mestrando em Políticas Públicas em Saúde, Escola Fiocruz de Governo, Brasília, DF, Brasil; pesquisador, Fundação de Empreendimentos Científicos e Tecnológicos, Brasília, Distrito Federal, Brasil. https://orcid.org/0000-0003-4693-3546. Email: akeni.lobo@gmail.com

2 Doutoranda em Saúde Pública, Universidade de Brasília, Brasília, Distrito Federal, Brasil. https://orcid.org/0000-00016662-0788. E-mail: indydymorais@gmail.com

${ }^{3}$ Mestrando em Ciências da Saúde, Escola Superior de Ciências da Saúde, Brasília, DF, Brasil. https://orcid.org/00000003-0965-9760. E-mail: velber35@gmail.com
} 
cidadãos no fornecimento de informações corretas, para o aprimoramento conjunto e contínuo da captação de dados, e a utilização correta dos dados por parte do Estado, sem ferir a privacidade dos cidadãos e melhorando a assistência à saúde da população.

Palavras-chave: Tecnologias da informação em saúde. Tecnologia da informação. Pandemias. Rede social.

\begin{abstract}
Objectives: to demonstrate how the use of health information technologies, by monitoring public access networks, contributes to the reduction of health conditions in pandemics, evaluating the use of technologies in international experiences and its potential applicability to Brazilian context. Methods: an integrative review was carried out with a survey of articles in the Scielo, Pubmed and Google Scholar databases. 21 articles and 11 different documents were selected, such as journalistic pieces, book chapters, reviews, among others. The articles were read and critically analyzed by two different researchers. A third researcher was responsible for interpreting the results. Results: the study demonstrated that there is a great worldwide trend towards the use of Information and Communication Technologies as tools to help fight pandemics, since there were several world examples that dealt with the use of technologies to control Covid-19, and that use depends on the evolution and improvement of technological tools and the legislation involved. In Brazil, there is great potential for evolution in this area, with increased data collection and use, assisting in public health strategies and actions. However, in all scenarios presented, there is a need for improvement and adjustments in the legislative scope - giving greater legal responsibility to privacy rights and social, with the expansion of the internet infrastructure, reaching the entire population and, mainly, with the greatest reliability of the data. Conclusion: based on the understanding of the scenario of use of Information and Communication Technologies in several countries and how the responses were made in their respective territories, a comparison was made with Brazil and it was noticed that the Brazilian technological capacity is limited in a way general. Sociocultural issues in Brazil stand out, because they are, at times, barriers that exist in the Brazilian scenario, and which must be discussed, aiming at a better understanding about the use of these technologies in public policies in order to reach the entire population. With that, it was seen that it is necessary the conscious and active participation of citizens in providing correct information, for the joint and continuous improvement of data collection, and the correct use of data by the State, without harming the privacy of citizens and improving health care for the population.
\end{abstract}

Keywords: Health information technologies. Information technology. Pandemics. Social network.

\title{
Resumen
}

Objetivos: demostrar cómo el uso de las tecnologías de la información en salud, mediante el monitoreo de las redes de acceso público, contribuye a la reducción de las condiciones de salud en las pandemias, evaluando el uso de las tecnologías en las experiencias internacionales y su aplicabilidad en la realidad brasileña. Metodología: Se realizó una revisión integradora con una encuesta de artículos en las bases de datos Scielo, Pubmed y Google Scholar. Se seleccionaron 21 artículos y 11 documentos diferentes, tales como piezas periodísticas, capítulos de libros, reseñas, entre otros. Los artículos fueron leídos y analizados críticamente por dos investigadores diferentes. Un tercer investigador se encargó de interpretar los resultados. Resultados: el estudio demostró que existe una gran tendencia mundial hacia el uso de las tecnologías de la información y las comunicaciones como 
herramientas para ayudar a enfrentar las pandemias, ya que hubo varios ejemplos mundiales que versaron sobre el uso de tecnologías para el control de Covid-19, y ese uso depende de la evolución y mejora de las herramientas tecnológicas y la legislación involucrada. En Brasil, existe un gran potencial de evolución en esta área, con una mayor recopilación y uso de datos, ayudando en las estrategias y acciones de salud pública. Sin embargo, en todos los escenarios presentados, existe la necesidad de mejoras y ajustes en el ámbito legislativo otorgando mayor responsabilidad legal a los derechos de privacidad - y social, con la expansión de la infraestructura de internet, llegando a toda la población y, principalmente, con la mayor confiabilidad. de los datos. Conclusión: a partir del entendimiento del escenario de uso de las tecnologías de la información y las comunicaciones en varios países y cómo se dieron las respuestas en sus respectivos territorios, se realizó una comparación con Brasil y se notó que la capacidad tecnológica brasileña es de alguna manera limitada general. Los temas socioculturales en Brasil se destacan, porque son, a veces, barreras que existen en el escenario brasileño, y que deben ser discutidas, buscando un mejor entendimiento sobre el uso de estas tecnologías en las políticas públicas para llegar a toda la población. Con ello, se vio que es necesaria la participación consciente y activa de la ciudadanía en la provisión de información correcta, para la mejora conjunta y continua de la recolección de datos, y el correcto uso de los datos por parte del Estado, sin dañar la privacidad de los ciudadanos y mejorar la atención de la salud de la población.

Palabras clave: Tecnologías de la información en salud. Tecnologías de la información. Pandemias. Red social.

\section{Introdução}

A pandemia do novo coronavírus (Covid-19) possui alcance mundial e números impressionantes de casos confirmados e óbitos, podendo ser retratada como uma das maiores contaminações da humanidade (1). Essa situação grave evidenciou problemas sociais como a desigualdade, o acesso aos serviços públicos e o alcance de políticas públicas, forçando o mundo a se reinventar e mudar as rotinas, as prioridades e as relações interpessoais.

Um dos grandes diferenciais em relação às pandemias anteriores se trata do nível de globalização da sociedade, que está cada vez mais conectada pelo uso da internet. Nesse momento, há uma produção incomparável de informações e conteúdos técnicos e científicos sobre a Covid-19, tornando o momento atual um grande marco na sociedade moderna.

A utilização das tecnologias vem como um meio de disseminar informações, usada por todos os segmentos sociais - governo, sociedade civil, entidades privadas ou entidades supranacionais - para disseminar informações em relação ao tema, sejam verdadeiras ou falsas (2). A sociedade globalizada precisa repensar a produção de aparatos e plataformas capazes de tornar possível a realização de procedimentos de monitoramento e controle em massa, com eficiência e praticidade (3). 
Há uma tendência mundial em utilizar as ferramentas digitais de informação que auxiliem no combate ao coronavírus. Diferentes países estão desenvolvendo estratégias para monitoramento e contenção à disseminação do vírus por meio de buscas ativas, com a participação de instituições privadas, da população e do próprio governo (4).

A utilização de dados tem sido cada vez mais aplicada às estratégias no âmbito da epidemiologia, visto que o manejo de big data se tem mostrado efetivo em colaborar com estudos e previsões para o enfrentamento adequado de futuras situações de saúde. $(2,5)$.

Nesse contexto, o artigo busca responder a seguinte pergunta de pesquisa: como as tecnologias da informação e comunicação em saúde podem auxiliar no combate a Covid-19 e quais as estratégias estão sendo utilizadas? O objetivo é demonstrar como o uso de tecnologias da informação em saúde por monitoramento de redes de acesso público contribui na redução de agravos das condições de saúde em pandemias, avaliando o uso das tecnologias nas experiências internacionais, além de apresentar como essas experiências internacionais podem ser adaptadas para a realidade brasileira.

\section{Metodologia}

Tratou-se de uma revisão integrativa (6) e o levantamento dos artigos foi realizado nas bases de dados Scielo, Pubmede Google Acadêmico. Usou-se como critério, a inclusão de artigos em português e inglês disponíveis integralmente de forma online e publicados entre janeiro e junho de 2020. Foram selecionados para o presente estudo 21 artigos e 11 documentos diversos, como peças jornalísticas, capítulos de livros, resenhas, entre outros.

Após a organização e sumarização dos artigos encontrados, foram realizadas leitura e análise crítica por dois pesquisadores distintos. Um terceiro pesquisador foi responsável pela interpretação dos resultados.

Todos os passos desta metodologia foram pensados e adaptados à realidade de publicações aceleradas de evidências científicas em momento de pandemia, por isso o pequeno recorte de tempo para os artigos selecionados. 


\section{Resultados e discussão}

\section{O contexto internacional}

Evidenciou-se a predominância de estudos (7-11) que demonstraram a eficácia dos métodos chineses de uso das tecnologias para o combate à pandemia (9).

O primeiro a ser destacado é o estudo de caso (4) sobre a estratégia chinesa de utilizar de ferramentas tecnológicas de detecção individual e a internet of things (loT), ou internet das coisas. Criou-se um banco de dados composto de algoritmos que analisa as diferentes realidades dentro de cenários em que haja qualquer interação entre objeto e sujeito, possibilitando o monitoramento de cidadãos, com controle direto da situação de saúde frente aos casos de coronavírus $(4,9)$. Foi utilizado um aplicativo de mensagens instantâneas popular na China, o Wechat, para realizar o controle de infectados e ser uma plataforma de informações confiáveis quanto à situação e o combate do coronavírus, informando também sobre as ações do governo $(4,9)$.

Dentre outras funcionalidades, o Wechat oferece o uso de QR code e a geolocalização para rastrear usuários que utilizam transportes públicos. Assim, ao entrar em algum modal da rede de transporte coletivo, o cidadão pode enviar informações sobre sua situação de saúde e seus dados pessoais ao governo. Com isso, as autoridades chinesas aumentaram a capacidade de rastreamento dos cidadãos com diagnóstico positivo, aperfeiçoando os procedimentos de contenção e freando a propagação da doença (12).

Já no estudo de Kummitha (4), também na China, analisou-se o uso de tecnologias inteligentes para o combate de pandemias, comparando ações tomadas por governos orientais e governos ocidentais. As tecnologias inteligentes fizeram parte do $12^{\circ}$ Plano Quinquenal, um planejamento político estratégico do governo chinês, com o compromisso de construir cidades inteligentes $(8,10)$.

Já o novo Plano Nacional de Urbanização na China, que entrou em vigor em 2014 e ainda está vigente, também prioriza não só a construção de novas cidades inteligentes, mas também a adaptação das cidades antigas ao novo modelo (11).

Com o aumento do êxodo rural e em resposta à crescente população urbana, o governo chinês assumiu um papel ativo, na qual as tecnologias foram desenvolvidas para acompanhar o crescimento exponencial e se tornar essencial na gestão, no controle e na governança das cidades povoadas por esses novos moradores. 
Existe uma estimativa que há aproximadamente 500 cidades inteligentes presentes na China (13). A cidade de Wuhan, uma das mais afetadas durante o surto de Covid-19, é uma delas e sua velocidade recuperativa demonstrou a eficiência dos métodos assumidos na cidade para o controle da disseminação (13).

Já o uso das tecnologias inteligentes nas democracias ocidentais, como na Europa, é mais burocrática, porém mais segura do ponto de vista da proteção de dados, tendo em vista a criação do Regulamento Geral sobre a Proteção de Dados (RGPD) da União Europeia e a descentralização das responsabilidades do poder decisório do Estado.

Os países europeus também apresentam vantagens como a facilidade de disseminação pela proximidade geográfica entre os países, além da aproximação política, econômica e social, o que facilita na condução articulada de políticas comuns. Com isso, as tecnologias podem ser amplamente utilizadas como forma de auxílio no combate à disseminação do coronavírus (4).

Em Milão, foram estabelecidos vários mecanismos inovadores para a adoção de tecnologias inteligentes, porém foi uma das cidades inteligentes mais afetadas em número de casos e óbitos na Itália. Sem esse controle, o número de infectados poderia ter se agravado, mas o que se viu foi uma ação considerável no controle de casos de infectados, feito a partir de um controle rigoroso (14). Existem outras cidades inteligentes que deram exemplo de controle, rastreamento e solução de casos de infectados como Berlim, na Alemanha, e Londres, na Inglaterra (4).

Os estudos têm demonstrado que as tecnologias inteligentes adotadas pelas cidades oferecem melhores condições de vida (15). O uso dessas tecnologias trouxe maior possibilidade de ação pelos Estados frente às pandemias, o que ofereceu certo alívio, porém, por outro lado, levantou discussões éticas relacionadas ao uso das informações pessoais para o monitoramento dos cidadãos, por parte da população e do legislativo desses países, diferentemente da China.

Outro estudo analisado foi o de Chen et al (7), sobre como envolver os cidadãos durante a pandemia por meio de mídias sociais. Chen et al apresentou uma outra visão sobre o envolvimento ativo dos cidadãos nas pautas públicas, empoderando os sujeitos como participantes e tomadores de decisões e aumentando as relações de confiança entre os Estados e o povo. 
A transparência proporcionada pelos governos que adotam estratégias participativas favorece o envolvimento dos cidadãos nas discussões de políticas públicas, fortalecendo as relações sociais; trazendo esclarecimentos e entendimento sobre as matérias discutidas; e proporcionando tomadas de decisões transparentes e mais justas às realidades $(16,17)$.

Em situações como a atual, de pandemia, cidadãos com experiências participativas colaboram ativamente nos levantamentos realizados pelos Estados, fornecendo informações relevantes e precisas para o controle das doenças.

Ainda no artigo de Chen et al (7), há relatos de uso de mídias sociais em situações de emergência, nem sempre diretamente ligadas a temas de saúde. Os autores destacam, por exemplo, que o Reino Unido usou o Twitter para o monitoramento de hashtags e menções e, assim, auxiliar na identificação de suspeitos durante ataques terroristas ocorridos em 2011. Na Indonésia, as ferramentas do Twitter foram utilizadas para a elaboração de estratégias de alerta à população durante o tsunami de 2012, aprimorando a prestação de serviços do Estado (7). É possível verificar que o uso de mídias sociais por parte dos governos quando em favor de estratégias públicas, são validadas para auxiliar em emergências.

Apesar dos aportes positivos, há um controle sobre o conteúdo e a forma de divulgação de informações e o uso da internet por parte do governo chinês. Nos últimos anos, a China definiu novas regras que estabelece o monitoramento das informações da população que podem ser consideradas ameaçadoras à segurança nacional do país, supervisionando as informações disponíveis online, sejam elas verdadeiras ou consideradas como boatos. O monitoramento permite que informações consideradas como violação às regras serão removidas e comunicadas às autoridades. Essas e outras ações estão presentes em diversos governos autoritários, onde os provedores de informações são impedidos de fazerem declarações de acordo com interesses do governo (18).

Bione (19) destaca que países como a China e os Estados Unidos trouxeram práticas de fiscalização ativa e invasiva com o uso de tecnologias de drones e câmeras de segurança para monitorar e avaliar os níveis de isolamento social, infringindo o direito à privacidade.

O autor conclui que os Estados, no afã de conter o aumento de casos de coronavírus, vêm adotando tecnologias para auxiliar no combate à epidemia e essas ações estão levantando questões sobre o uso de informações pessoais e a proteção de dados (19). 
No Ocidente, as estratégias de países como Itália, França, Letônia, Suíça e o Reino Unido incluíam aplicativos próprios para o rastreamento, contenção e ampliação das informações sobre o Covid-19. A tecnologia utilizada nos modelos europeus é o rastreamento automatizado de contato pessoal, envolvendo o uso de smartphones para detectar quando as pessoas ficam próximas por um período suficiente para que haja um risco significativo de contágio. Caso alguma delas seja diagnosticada com a doença, as pessoas envolvidas podem ser notificadas.

Para a utilização do rastreamento, é necessário acessar a application programming interface, (API, sigla em inglês), que no português se traduz em interface de programação de aplicativos dos smartphones. É uma ferramenta de software que dá acesso a alguns recursos dos sistemas operacionais móveis IOS e Android (20).

A França e o Reino Unido estão, até o presente momento, testando e aprimorando suas versões para ampliar a funcionabilidade da tecnologia de rastreamento. $O$ modelo escolhido por esses países é uma forma centralizada da captação e gestão de dados para fornecer informações aos cientistas, possibilitando um direcionamento das estratégias e uma maior precisão nas ações para a prevenção do contágio. O uso desse modelo centralizado não se enquadra nas regras de empresas pela proibição da coleta de dados por localização, o que, para alguns especialistas, pode ser visto como invasão de privacidade (20).

Na Itália, Letônia e Suíça, que desenvolveram aplicativos em parceria com a Apple e a Google obtiveram uma melhor proteção do anonimato e da privacidade dos usuários com o uso de tecnologia descentralizada baseada no handshakes, que é a conectividade utilizando o bluetooth, uma conexão por proximidade (20).

A relação entre os modelos centralizado e descentralizado parte de um mesmo princípio, no qual sujeitos cujos dispositivos contam com tecnologias de código-chave se encontram e um deles, estando infectado com o coronavírus, atualiza seu status de saúde por meio dessa tecnologia (21).

No modelo centralizado, após esse input de dados, o telefone fornece ID próprio, anônimo, e os códigos coletados de outros telefones para o banco de dados centralizado. Depois, o servidor usa o banco de dados para fazer a correspondência de contatos, analisando os riscos de contágio entre as pessoas que estiveram próximas ao infectado e enviar os alertas em caso necessário (21). Já no modelo descentralizado, o telefone fornece apenas o ID próprio e anônimo para o banco de dados centralizado. O celular faz o download 
do banco de dados para fazer a correspondência de contatos, analisando os riscos de contágio e enviando os alertas em caso de possível contaminação (21).

A diferença entre os modelos está na relação dos atores; no modelo descentralizado existe um poder de decisão e consentimento do sujeito não infectado para que tome medidas quanto à possibilidade de contágio, sem a necessidade do controle de um servidor, aumentando a condição de segurança de dados e da privacidade do usuário.

\section{O contexto brasileiro no uso das tecnologias de informação e comunicação (TIC)}

O uso de informações e dados para estratégias de combate ao vírus está diretamente relacionado aos aspectos sociais que envolvem o desenvolvimento social da população em um contexto epidemiológico. Outros fatores que influenciam é o acesso a equipamentos de qualidade e à internet, além da experiência que permite às pessoas se familiarizarem o suficiente com as tecnologias disponíveis para obter algum tipo de benefício em sua utilização (2).

Nesse contexto, Beaunoyer, Dupéré e Guitton (2) discutem como a pandemia do coronavírus potencializou as desigualdades digitais, e como essas desigualdades tiveram impacto sobre os determinantes da saúde.

As questões sociais e culturais são fatores de grande importância para o debate, visto que as particularidades da heterogeneidade social do Brasil fazem com que enfrente desafios diferentes da maioria dos outros países. A relação entre as desigualdades digitais e sociais é também considerada como um fator resultante do contexto socioeconômico (5).

O IBGE mostrou que, em 2018, o Brasil possuiu 166 milhões de usuários de internet, o que significou um aumento de usuários em comparação com 2016 e 2017. O maior crescimento aconteceu entre os idosos com mais de 60 anos, mas todas as outras categorias etárias também tiveram crescimento no número de usuários(8). Ainda que não se tenha oficialmente os motivos para o aumento do número de idosos com acesso à internet, inferese que houve um aumento do número de pessoas que se tornaram idosas e que já estavam mais habituadas a utilizarem telefones celulares para se relacionar em suas redes sociais.

Porém, os aspectos de analfabetismo digital ainda estão presentes em grande parte dos usuários, que dependem quase sempre de auxílio para o desenvolvimento de atividades comuns nos celulares o que se tornam barreiras para o uso mais aprofundado dos serviços fornecidos pelos aparelhos. 
É notório o aumento do uso das tecnologias digitais, visto que se tornaram um dos principais meios de comunicação e interações entre cidadãos e 0 Estado. Consequentemente, a limitação do acesso às tecnologias digitais promove uma limitação aos serviços, o que impacta na saúde (21).

Quando comparados ao crescimento da cobertura da internet, a pesquisa do IBGE demonstra que aumentou o número de usuários nas cidades das cinco regiões do país e em suas áreas rurais. Segundo a pesquisa, existem mais de quatro milhões de residências rurais do país, onde ao menos um morador da residência tem acesso à internet. Porém, ainda são considerados números baixos, pois apenas $41 \%$ de toda a população rural do país tem algum acesso à internet, enquanto entre a população urbana essa proporção é de mais de $80 \%(22)$.

Quanto à qualidade do acesso à internet, de acordo com a Anatel, durante o período de janeiro a dezembro de 2019 , o percentual de cumprimento de metas do serviço alcançou $77,1 \%$ no território nacional, demonstrando que não há cobertura total, nem prestação de serviço com desempenho apropriado (23).

Já em relação ao custo, os dados demostraram que a desigualdade social favoreceu a inserção da internet nas camadas sociais mais abastadas, quando comparados os valores gastos pelo uso da internet: em 2010, a média cobrada pelos provedores de internet era de $\mathrm{R} \$ 21,18$ por 1 megabit per second (Mbps); em 2017, a média chegou a $\mathrm{R} \$ 4,62$ (23).

As evidências apontam que há diferença da abrangência de acesso à internet por região geográfica e por população urbana e rural. Essa diferença demonstra os problemas estruturais no Brasil, contudo, há a percepção promissora para o desenvolvimento de ferramentas e planos epidemiológicos contingenciais, que alcancem diferentes parcelas da população brasileira.

\section{Dados sensíveis e privacidade no Brasil}

Outro ponto de discussão é a proteção de dados pessoais no uso das TICs. No Brasil, com a publicação da Lei no 13.709/2018, chamada de Lei Geral de Proteção de Dados (LGPD) (24), apresenta-se um novo paradigma sobre a proteção de dados pessoais, especialmente importantes em um contexto pandêmico. Com o cenário favorável a discussões sobre essa temática, o país se viu obrigado a regulamentar os dispostos na nova 
lei, considerando os fatores que se associam aos dados pessoais e a preservação do indivíduo com o desenvolvimento de políticas públicas de saúde (24).

No recém-lançado livro Os dados e o vírus: Pandemia, proteção de dados e democracia (19), os autores discutem sobre a LGPD, em que a considera como uma verdadeira mudança de paradigma no que diz respeito à proteção de dados pessoais, considerando ser um avanço desejável e amplamente aguardado diante o atual cenário epidemiológico.

De acordo com a LGPD(24), o artigo $7^{\circ}$ define que o tratamento de dados pessoais somente poderá ser realizado em algumas hipóteses, sendo uma delas "[...] a tutela da saúde, exclusivamente, em procedimento realizado por profissionais de saúde, serviços de saúde ou autoridade sanitária". Em complemento, o artigo 10² (24) dispõe sobre o que é considerado "o legítimo interesse do controlador", que poderá manusear os dados pessoais caso tenha finalidades legítimas devidamente fundamentadas em fatos concretos, que incluem, mas não se limitam a:

I - apoio e promoção de atividades do controlador; e

II - proteção, em relação ao titular, do exercício regular de seus direitos ou prestação de serviços que o beneficiem, respeitadas as legítimas expectativas dele e os direitos e liberdades fundamentais, nos termos desta Lei.

$\S 1^{\circ}$ Quando o tratamento for baseado no legítimo interesse do controlador, somente os dados pessoais estritamente necessários para a finalidade pretendida poderão ser tratados.

$\S 2^{0}$ O controlador deverá adotar medidas para garantir a transparência do tratamento de dados baseado em seu legítimo interesse.

$\S 3^{\circ}$ A autoridade nacional poderá solicitar ao controlador relatório de impacto à proteção de dados pessoais, quando o tratamento tiver como fundamento seu interesse legítimo, observados os segredos comercial e industrial". (24)

Por fim, dentre os principais pontos da LGPD (24) que tratam da temática que envolve a saúde e o uso dos dados, está o artigo 11ํㅜ que fala sobre as hipóteses do tratamento de dados pessoais sensíveis:

"I - quando o titular ou seu responsável legal consentir, de forma específica e destacada, para finalidades específicas;

II - sem fornecimento de consentimento do titular, nas hipóteses em que for indispensável para:

[...]

f) tutela da saúde, exclusivamente, em procedimento realizado por profissionais de saúde, serviços de saúde ou autoridade sanitária". (24) 
A partir de uma visão jurídica centrada nos aspectos do uso de dados sensíveis em meio a crises de saúde pública, a discussão trazida pelo artigo 11ำ trata de pontos da LGPD no âmbito da saúde da população (25) demonstra que o livre consentimento nas situações de tratamento de dados está previsto na legislação.

Assim, em situações de exceção, quando a dispensa de consentimento ocorre em casos de proteção à vida ou tutela da saúde, em procedimento realizado por profissionais da saúde; em serviços de saúde; por autoridades públicas sanitárias; ou, ainda, no caso de estudo por órgãos de pesquisa, a utilização de dados pessoais está prevista na LGPD. Portanto, essas possibilidades, mesmo que sejam em situações de exceção, entram na discussão pela questão do uso das informações sem o consentimento do titular-usuário, o que pode trazer incertezas quanto à privacidade de dados.

A LGPD traz em seu texto a vedação do uso comercial dos dados pessoais de saúde sem autorização expressa do usuário, como por exemplo por meio do Termo de Condições e Autorização para os controladores de dados. Porém, para fins de políticas públicas e pesquisas, não é necessária a autorização.

Quando se trata de benefício dos interesses dos titulares de dados, pode ser uma brecha para a vigilância e má utilização dos dados, o que iria de encontro ao que está ao previsto em lei. A preocupação com a utilização indiscriminada e sem o consentimento do titular é considerável, visto o potencial do uso de dados sensíveis em massa (26).

Os dados sensíveis são dados pessoais que estão sujeitos a condições de tratamento específicas: dados pessoais que revelem a origem racial ou étnica, opiniões políticas e convicções religiosas ou filosóficas; filiação sindical; dados genéticos, dados biométricos tratados simplesmente para identificar um ser humano; dados relacionados com a saúde; dados relativos à vida sexual ou orientação sexual (27). Assim, devido à amplitude de conhecimento sobre a população, suas preferências, rotinas e perfis, o uso dessas informações torna as massas fragilizadas aos controladores.

No caso da realização de estudos na área da saúde pública que realizem pesquisas em saúde, existe uma limitação para o uso de dados, pois os órgãos podem usar as bases de dados, porém devem ser tratados exclusivamente no próprio órgão e com finalidade estrita às realizações dos estudos, garantindo sempre que possível o anonimato, respeitando os aspectos éticos. 
Com isso, a discussão dos impactos da LGPD na população em relação à área da saúde exige evidências e discussões para se fazer uma análise sobre suas consequências nas políticas públicas e na privacidade do cidadão (25). Deve-se ressaltar a importância da participação de diferentes atores, como o Ministério Público e sociedade civil organizada, que são capazes de realizar a manutenção do entendimento da privacidade e do uso dos dados. Esses atores serão capazes de responsabilizar os gestores públicos, cobrando ou punindo por excessos decorrentes de ações que extrapolem os direitos fundamentais nesse contexto de pandemia (26).

Em paralelo às ações político e científicas, visando a solução da discussão sobre privacidade de dados, o Banco Interamericano de Desenvolvimento (BID) desenvolveu o aplicativo David-19 em parceria com Everis, IOVlabs e World Data, que são empresas multinacionais de tecnologia, para proporcionar uma ferramenta de monitoramento diante da crise. Esse aplicativo pode ser uma solução capaz de auxiliar governos locais, compartilhando dados sobre o coronavírus sem expor a privacidade dos usuários, pois conta com tecnologia blockchain, que pode ser entendida como o uso de protocolo de segurança permitindo aos cidadãos o compartilhamento de dados sem expor a privacidade (11).

Assim, a heterogeneidade do Estado brasileiro impede uma avaliação taxativa sobre a relação entre o uso das TICs e o auxílio efetivo ao combate às pandemias. Porém, como um dos principais pilares da saúde é a universalidade, deve-se considerar que o país ainda enfrenta limitações tanto tecnológicas, quanto legislativas

\section{Conclusão}

Este estudo discutiu sobre as diversas estratégias de países que utilizaram o apoio das diferentes tecnologias da informação em saúde de massa para o enfrentamento de pandemias, controle e prevenção de situações de desastres naturais, com destaque para China, Itália, Estados Unidos Letônia, Alemanha e Inglaterra.

No Brasil, ainda que possua capacidade tecnológica limitada e conte com diversidade sociocultural e política, poderia se valer das diferentes experiências internacionais para uma melhor elaboração de planos estratégicos no enfrentamento de pandemias, com a devida adaptação à dinâmica populacional e estrutura do Estado.

Percebe-se, a partir da metodologia, que o uso de dados pessoais se mostra uma boa aliada no enfrentamento da pandemia, com a criação de parcerias entre o governo e 
empresas de comunicação digital, provedores de internet, empresas detentoras de mídias digitais de comunicação rápida online.

Depreende-se das evidências identificadas nos estudos que as opiniões convergem na questão das desigualdades digitais como um fator de aprofundamento da crise epidemiológica no mundo. Essa desigualdade digital merece destaque por refletir a dimensão das desigualdades sociais existentes no mundo, não sendo diferente no Brasil.

Portanto, no contexto brasileiro, pode-se perceber possíveis soluções para o aperfeiçoamento do enfrentamento às pandemias, com a maior participação da população; melhor distribuição de renda; o aumento no acesso às condições básicas de educação e saneamento; e acesso à internet, cultura e informação. O Estado também deve priorizar as políticas de desenvolvimento tecnológico, com a intersetorialidade na educação, na economia e na força de trabalho, aumentando os investimentos nessas áreas e promovendo capacitações diretivas para alcançar as metas governamentais.

Outras ações do Estado, em suas diferentes esferas, é o desenvolvimento de políticas públicas que promovam acesso à internet para a população de baixa renda, como uma extensão dos programas assistenciais do governo, fornecendo internet aos cidadãos cadastrados nos programas de renda. Seriam fornecidos por empresas de comunicação consolidadas, nas diferentes regiões do país. Assim, o Estado pode promover uma maior inclusão digital, além de aumentar as possibilidades de atualização cadastral desses usuários.

A discussão merece ainda um maior aprofundamento sobre as questões socioculturais que permeiam os determinantes sociais da saúde, a fim de definir as políticas de uso dos dados que necessitem da colaboração ativa da sociedade para a efetivação de ações eficazes no combate às pandemias no país.

\section{Referências}

1. World Health Organization. Coronavirus disease (COVID-19) pandemic [Internet]. 2020 [cited 2020 Jun 26]. Available from: https://www.who.int/emergencies/diseases/novelcoronavirus-2019?gclid=Cj0KCQjwudb3BRC9ARIsAEavUvoyyLhZt1v2sVvNWUJVjzHZwU3Yq-Ko0LRFXkCHKpwvpVe7EuLfzcaAg76EALw_wcB

2. Beaunoyer E, Dupéré S, Guitton MJ. COVID-19 and digital inequalities: Reciprocal impacts and mitigation strategies. Comput Human Behav. 2020 Oct 1;111:106424. 
3. Santos M. Por uma outra globalização: do pensamento único à consciência universal. $4^{\underline{a}}$ Edição. São Paulo: Record; 2000

4. Kummitha RKR. Smart technologies for fighting pandemics: The techno- and humandriven approaches in controlling the virus transmission. Government Information Quarterly. Elsevier Ltd; 2020. p. 101481.

5. Robinson L, Cotten SR, Ono H, Quan-Haase A, Mesch G, Chen W, et al. Digital inequalities and why they matter. Inf Commun Soc [Internet]. 2015 May 4 [cited 2020 Jun 24];18(5):569-82. Available from:

https://www.tandfonline.com/doi/abs/10.1080/1369118X.2015.1012532

6. Costa KB, Silva LM, Ogata MN. A judicialização da saúde e o Sistema Único de Saúde: revisão integrativa. Cad Ibero-Americanos Direito Sanitário [Internet]. 2020 [cited 2020 Aug 5]. Available from:

https://www.cadernos.prodisa.fiocruz.br/index.php/cadernos/article/view/635/741

7. Chen Q, Min C, Zhang W, Wang G, Ma X, Evans R. Unpacking the black box: How to promote citizen engagement through government social media during the COVID-19 crisis. Comput Human Behav [Internet]. 2020 Sep 1 [cited 2020 Jun 24];110:106380. Available from: /pmc/articles/PMC7151317/?report=abstract

8. Chen L, Naughton B. An institutionalized policy-making mechanism: China's return to techno-industrial policy. Res Policy. 2016 Dec 1;45(10):2138-52.

9. Kamel Boulos MN, Al-Shorbaji NM. On the Internet of Things, smart cities and the WHO Healthy Cities [Internet]. Vol. 13, International Journal of Health Geographics. BioMed Central Ltd.; 2014 [cited 2020 Jun 24]. p. 10. Available from: http://ijhealthgeographics.biomedcentral.com/articles/10.1186/1476-072X-13-10

10. Liu FC, Simon DF, Sun YT, Cao C. China's innovation policies: Evolution, institutional structure, and trajectory. Res Policy. 2011 Sep 1;40(7):917-31.

11. Zhu S, Li D, Feng $\mathrm{H}$. Is smart city resilient? Evidence from China. Sustain Cities Soc. 2019 Oct 1;50:101636.

12. Cury ME. Como a China usou o WeChat para conter a covid-19 - e vigiar as notícias. Exame [Internet]. 2020 Apr 4 [cited 2020 Jun 26]. Available from:

https://exame.com/tecnologia/como-a-china-usou-o-wechat-para-conter-a-covid-19-e-vigiaras-noticias/

13. Mak R. Breakingviews - Wuhan virus will shape China's smart city vision [Internet]. NASDAQ. 2020 [cited 2020 Jun 24]. Available from:

https://www.nasdaq.com/articles/breakingviews-wuhan-virus-will-shape-chinas-smart-cityvision-2020-01-24

14. Carè S, Trotta A, Carè R, Rizzello A. Crowdfunding for the development of smart cities. Bus Horiz. 2018 Jul 1;61(4):501-9. 
15. Kamel Boulos MN, Resch B, Crowley DN, Breslin JG, Sohn G, Burtner R, et al. Crowdsourcing, citizen sensing and sensor web technologies for public and environmental health surveillance and crisis management: Trends, OGC standards and application examples [Internet]. Vol. 10, International Journal of Health Geographics. Int J Health Geogr; 2011 [cited 2020 Jun 24]. Available from:

https://pubmed.ncbi.nlm.nih.gov/22188675/

16. Agostino D, Arnaboldi M. A Measurement Framework for Assessing the Contribution of Social Media to Public Engagement: An empirical analysis on Facebook. Public Manag Rev [Internet]. 2016 Oct 20 [cited 2020 Jun 26];18(9):1289-307. Available from: https://www.tandfonline.com/doi/abs/10.1080/14719037.2015.1100320

17. Taylor M, Kent ML. Dialogic Engagement: Clarifying Foundational Concepts. J Public Relations Res [Internet]. 2014 Oct 20 [cited 2020 Jun 26];26(5):384-98. Available from: http://www.tandfonline.com/doi/abs/10.1080/1062726X.2014.956106

18. Novas regras vão aumentar controle da internet na China [Internet]. Agência Brasil. 2020 [cited 2020 Jun 24]. Available from:

https://agenciabrasil.ebc.com.br/internacional/noticia/2020-03/novas-regras-vao-aumentarcontrole-da-internet-na-china

19. Bioni BR, Zanatta RAF, Rielli M, Vergili G, Favaro I et al. Os dados e o vírus:

Pandemia, proteção de dados e democracia. São Paulo: Reticências Creative Design Studio; 2020.

20. Kelion L. Coronavirus: France's virus-tracing app "off to a good start." BBC News [Internet]. 2020 [cited 2020 Jun 24]. Available from: https://www.bbc.com/news/technology52905448

21. Government of South Australia. Digital Technology A Health Lens [Internet]. 2009 [cited 2020 Jun 24]. Available from:

https://www.sahealth.sa.gov.au/wps/wcm/connect/0f50cc8043aecfaeb519fded1a914d95/DI GITAL+TECHNOLOGY+REPORT+FINAL.pdf?MOD=AJPERES\&CACHEID=0f50cc8043ae cfaeb519fded1a914d95

22. IBGE. Acesso à internet e à televisão e posse de telefone móvel celular para uso pessoal 2018 [Internet]. 2020 [cited 2020 Jun 26]. Available from:

https://biblioteca.ibge.gov.br/index.php/biblioteca-catalogo?view=detalhes\&id=2101705

23. Anatel. Relatório de acompanhamento do setor de telecomunicações: Serviço de Comunicação Multimídia - Banda Larga Fixa. 2018;32. Available from: http://www.anatel.gov.br/dados/relatorios-de-acompanhamento/2018

24. Brasil. Lei $n^{\circ} 13.709$, de 14 de agosto de 2018 [Internet]. 2018. Available from: http://www.planalto.gov.br/ccivil_03/_Ato2015-2018/2018/Lei/L13709.htm 
25. Peck P. LGPD e saúde: os fins justificam os meios? - LGPD - Lei Geral de Proteção de Dados Pessoais [Internet]. Serpro. 2019 [cited 2020 Jun 24]. Available from:

https://www.serpro.gov.br/lgpd/noticias/2019/paciente-no-comando-lgpd-dados-sensiveissaude

26. Fantini LMC. Coronavírus - SUS: Aspectos Relevantes da Privacidade e Proteção de Dados e Tecnologia de Vigilância. In: Bioni BR, Zanatta RAF, Rielli M, Vergili G, Favaro I et al. Os dados e o Vírus: Pandemia, proteção de dados e democracia. São Paulo:

Reticências Creative Design Studio; 2020. 136-144.

27. O Parlamento Europeu e o Conselho da União Europeia. Regulamento (UE) 2016/679 do Parlamento Europeu e do Conselho, de 27 de abril de 2016, relativo à proteção das pessoas singulares no que diz respeito ao tratamento de dados pessoais e à livre circulação desses dados e que revoga a Diretiva 95/46/CE (Regulamento Geral sobre a Proteção de Dados) [Internet]. 2016 [cited 2020 Jun 24]. Available from: https://eurlex.europa.eu/legal-content/PT/TXT/?uri=celex\%3A32016R0679

\section{Colaboradores}

Coelho AL contribuiu com a concepção/desenho do artigo, redação, revisão crítica e aprovação da versão final do artigo. Morais IA contribuiu para a concepção/desenho do artigo, redação do artigo, revisão crítica e aprovação da versão final do artigo. Rosa WVS contribuiu com a redação, revisão crítica e aprovação da versão final do artigo. pandemia do Covid-19 no Brasil. Cadernos Ibero-Americanos de Direito Sanitário. 2020 jul./set.; 9(3): $183-199$. 